\title{
First Human Infection with Avian Influenza H9N2 - Guangdong Province, China, 2020
}

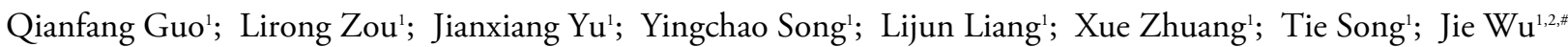

A case of avian influenza H9N2 was detected in a human in Zhuhai City, Guangdong Province through routine influenza surveillance on March 30, 2020. The patient was a 3-year-old child who developed fever and cough on March 22. A pharyngeal swab was collected on March 23, and the novel coronavirus nucleic acid test was negative. On March 26, the pharyngeal swab was sent to Zhuhai CDC for influenza virus tests and was found to be $\mathrm{H} 9$ influenza $\mathrm{A}$ positive, and on March 30, Guangdong Provincial CDC reexamined the pharyngeal swabs as positive for influenza $A$ (H9N2). On March 28, the patient was hospitalized for isolation treatment, and oseltamivir and other antiviral drugs were given in the hospital. A pharyngeal swab was collected again for reexamination on March 28, and the H9 test was negative. Epidemiological investigation found that the patient had been exposed to chickens and ducks raised by her grandparents before onset of illness. Samples taken from 5 close contacts and the environment of her grandparents' home all tested negative for the $\mathrm{H} 9$ nucleic acid.

The pharyngeal swab samples collected on March 23 were inoculated into an 11-day-old specific pathogen free (SPF) chicken embryo in Guangdong Provincial CDC. On April 7, the chicken embryo's allantoic fluid was collected and identified as $\mathrm{A}(\mathrm{H} 9 \mathrm{~N} 2)$ positive, with the strain named A/Guangdong/Zhuhai/20SF8034/ 2020(H9N2). The isolated strain was sequenced and all 8 virus sequences of $\mathrm{A}(\mathrm{H} 9 \mathrm{~N} 2)$ were obtained. The 8 gene sequences have been uploaded to GISAID (GISAID accession numbers: EPI1738873EPI1738880). Basic local alignment search tool (BLAST) in GenBank showed that the hemagglutinin (HA) gene of this virus was most similar with A/duck/China/d4/2018(H9N2) that was isolated from Guangdong Province, China, with a sequence similarity of $99.06 \%$. When compared with the HA genes of $36 \mathrm{H} 9 \mathrm{~N} 2$ virus isolates from human infection cases (searched from GISAID), the sequence similarity with A/Hunan/34179/2018 was the highest (98.4\%). BLAST in GenBank showed that the NA gene of this virus was most similar with $\mathrm{A} /$ chicken/Shanghai/
$07 / 2018$ (H9N2) at $99.06 \%$. Comparison with the NA genes of $36 \mathrm{H} 9 \mathrm{~N} 2$ virus isolates from human infection cases, sequence similarity was highest at $98.4 \%$ with A/Fujian-Sanyuan/2881/2019(H9N2).

Phylogenetic analysis of HA and NA was carried with reference strains including the World Health Organization (WHO) recommended pre-pandemic vaccine strains (1), similar $\mathrm{H} 9 \mathrm{~N} 2$ strain sequences from GenBank, and all human avian influenza virus sequences from GISAID. HA phylogenetic tree analysis showed the virus belong to the Y280/G9 clade and shared the same branch with $\mathrm{A} /$ duck/China/ D4/2018(H9N2) from ducks and A/Hunan/34179/ 2018(H9N2) from humans (Figure 1). NA Phylogenetic tree analysis showed the virus belongs to the Y280/G9 clade and was clustered with A/chicken/ Shanghai/07/2018(H9N2), A/chicken/China/1103/ 2019(H9N2), and A/chicken/China/71/2019(H9N2) (2) (Figure 2). BLAST analysis of the internal gene segments (MP, NP, NS, PA, PB1, PB2) of the isolated strain found that these 6 segments all had the highest similarity with avian-derived A (H9N2) with molecular markers of avian-like viruses, suggesting that the risk of human infection was not higher than that of previous $\mathrm{H} 9 \mathrm{~N} 2$ avian influenza viruses.

H9N2 is the most common subtype of avian influenza in poultry, and human infection is relatively rare (3-4). A total of 11 cases of H9N2 have been reported since 2010 in Guangdong Province, all of which were mild cases and have recovered. The case of H9N2 detected this time is an occasional case of human infection with $\mathrm{H} 9 \mathrm{~N} 2$ avian influenza virus. We should continue to strengthen the risk monitoring and early warning of avian influenza on the basis of the existing surveillance of avian influenza.

Fundings: This study was supported by the Key Research and Development Program of Guangdong Province (2019B111103001), and Natural Science Foundation of Guangdong Province (2019A1515 012121).

doi: $10.46234 / \mathrm{ccdcw} 2020.147$

\#Corresponding author: Jie Wu, 771276998@qq.com. 


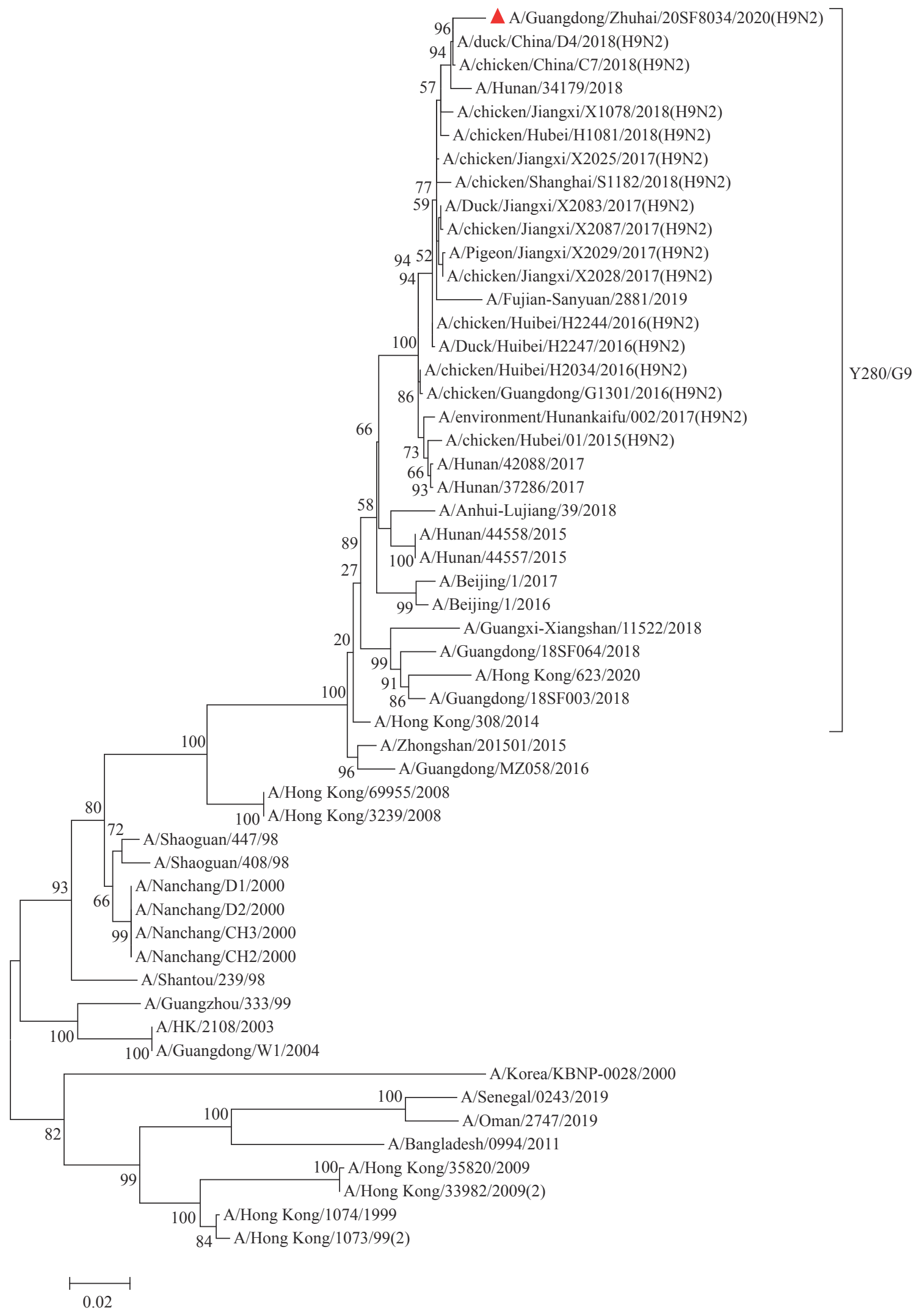

FIGURE 1. Phylogenetic tree based on HA gene sequences of the isolated virus strain A/Guangdong/Zhuhai/20SF8034/ 2020(H9N2) and the reference strains using the maximum likelihood method. A red solid triangle marks the location of A/Guangdong/Zhuhai/20SF8034/2020(H9N2). 


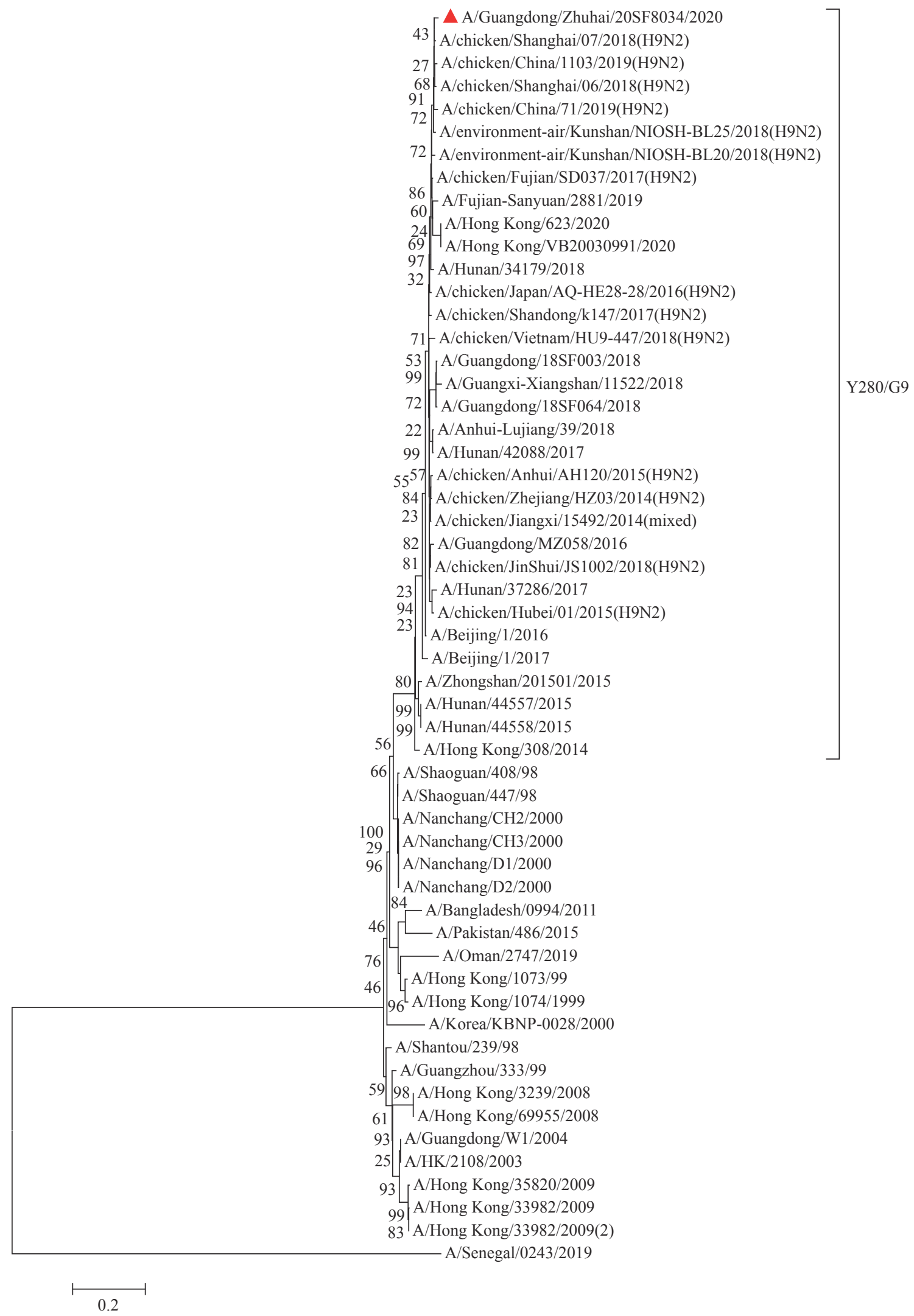

FIGURE 2. Phylogenetic tree based on neuraminidase (NA) gene sequences of the isolated virus strain $A / G u a n g d o n g / Z h u h a i /$ 20SF8034/2020 (H9N2) and the reference strains using the maximum likelihood method. A red solid triangle marked the location of A/Guangdong/Zhuhai/20SF8034/2020(H9N2). 
${ }^{1}$ Guangdong Provincial Center of Disease Control and Prevention, Guangzhou, Guangdong, China; ${ }^{2}$ Southern Medical University, School of Public Health, Guangzhou, Guangdong, China.

Submitted: June 24, 2020; Accepted: July 02, 2020

\section{REFERENCES}

1. World Health Organization. Candidate vaccine viruses for $\mathrm{A}(\mathrm{H} 9 \mathrm{~N} 2)$. https://www.who.int/influenza/vaccines/virus/candidates_reagents/a_h9n 2/en/.[2020-05-21].

2. Wu YH, Lin JS, Yang SH, Xie Y, Wang M, Chen XQ, et al. The molecular characteristics of avian influenza viruses (H9N2) derived from air samples in live poultry markets. Infect Genet Evol 2018;60:191 - 6 . http://dx.doi.org/10.1016/j.meegid.2018.01.009.

3. Li C, Wang SG, Bing GX, Carter RA, Wang ZJ, Wang JL, et al. Genetic evolution of influenza $\mathrm{H} 9 \mathrm{~N} 2$ viruses isolated from various hosts in China from 1994 to 2013. Emerg Microbes Infect 2017;6(1):e106. http://dx.doi.org/10.1038/emi.2017.94.

4. Xia J, Cui JQ, He X, Liu YY, Yao KC, Cao SJ, et al. Genetic and antigenic evolution of $\mathrm{H} 9 \mathrm{~N} 2$ subtype avian influenza virus in domestic chickens in southwestern China, 2013-2016. PLoS One 2017;12(2): e0171564. http://dx.doi.org/10.1371/journal.pone.0171564. 